\title{
Prehabilitación en el paciente con sarcopenia candidato a procedimientos quirúrgicos
}

\author{
Prehabilitation in patients with sarcopenia who are candidates for surgical \\ procedures
}

Pré-habilitação no paciente com sarcopenia candidato a procedimentos cirúrgicos

Jessika Cadavid Sierra*1, José Eduardo de Aguilar-Nascimento², Diana B. Dock-Nascimento³.

Recibido: 6 de agosto de 2020. Aceptado para publicación: 27 de octubre de 2020.

Publicado en línea: 14 de noviembre de 2020.

https://doi.org/10.35454/rncm.v4n1.193

\begin{abstract}
Resumen
El envejecimiento es un proceso natural en el ser humano que trae consigo cambios fisiológicos, morfológicos, bioquímicos y psicológicos. A medida que van aumentando los años, estas modificaciones se traducen en una mayor vulnerabilidad para el desarrollo de enfermedades como la sarcopenia, que implican una disminución de la capacidad funcional, pérdida de masa y fuerza muscular, y mayor fragilidad. Los procedimientos quirúrgicos, cada vez más comunes en la población geriátrica, representan un gran desafío para el personal médico en la medida en que esta población requiere de cuidados individualizados y de una atención multidimensional enfocada en diferentes aspectos, que van desde la salud física y emocional hasta aspectos económicos, sociales y culturales. En los pacientes sarcopénicos candidatos a procedimientos quirúrgicos son necesarias intervenciones preoperatorias que optimicen su salud y aceleren su recuperación posoperatoria, y disminuyan el riesgo de complicaciones y mortalidad. Esta revisión examina cómo la prehabilitación representa una oportunidad para mejorar la capacidad funcional de los pacientes y para prepararlos para soportar un evento estresante como lo es una cirugía, haciendo más digno su curso posoperatorio y mejorando su calidad de vida a corto y largo plazo.
\end{abstract}

Palabras clave: sarcopenia, cirugía general, atención perioperativa, fragilidad, ejercicio físico.

\begin{abstract}
Aging is a natural process in humans that brings physiological, morphological, biochemical, and psychological changes. As age increases, these modifications translate into increased vulnerability for diseases like sarcopenia, which implies a decrease in functional capacity, loss of muscle mass and strength, and greater fragility. Surgical procedures are becoming increasingly common in the geriatric population, and represent a major challenge for the medical staff due to the fact that this population requires and individualized and multidimensional care focused on different aspects, ranging from physical and emotional health, to economic, social and cultural aspects. In sarcopenic patients who are candidates for surgical procedures, preoperative interventions are necessary to optimize their well-being, accelerate their postoperative recovery, and reduce the risk of complications and death. This review evaluates how prehabilitation represents an opportunity to improve the functional capacity of patients with sarcopenia and prepares them to withstand a stressful event such as surgery, making their postoperative course more dignified, and improving their short and long-term quality of life.
\end{abstract}

Keywords: Sarcopenia; General Surgery; Perioperative Care; Frailty; Physical exercise.

\section{Resumo}

O envelhecimento é um processo natural do ser humano que acarreta mudanças fisiológicas, morfológicas, bioquímicas e psicológicas. Com o passar dos anos, essas modificações se traduzem em maior risco de desenvolver doenças como a sarcopenia, que implica na diminuição da capacidade funcional, perda de massa e força muscular e maior fragilidade. Os procedimentos cirúrgicos, cada vez mais comuns na população geriátrica, representam um grande desafio para o pessoal médico na medida em que esta população requer cuidados individualizados e de uma atenção multidisciplinar focada em diversos aspectos que vão desde a saúde física e emocional até aspectos econômicos, sociais e culturais. Nos pacientes sarcopênicos candidatos a procedimentos cirúrgicos, as intervenções pré-operatórias são necessárias para otimizar sua saúde e acelerar a recuperação pós-operatória, reduzindo o risco de complicações e mortalidade.

Esta revisão examina como a pré-habilitação representa uma oportunidade para melhorar a capacidade funcional dos pacientes e prepará-los para suportar um evento estressante como a cirurgia, tornando seu pós-operatório mais digno e melhorando sua qualidade de vida em curto e longo prazo.

Palavras-chave: sarcopenia, cirurgia geral, cuidados peri-operatórios, fragilidade, exercício físico. 
Cepain Institución Prestadora de Salud. Medellín, Colombia.

*Correspondencia: Jessika Cadavid Sierra. jessikacadavid.sierra@gmail.com

\section{INTRODUCCIÓN}

El proceso de envejecimiento de la población mundial está siendo cada vez más rápido. Según la Organización Mundial de la Salud (OMS), se estima que entre 2015 y 2050, la proporción de la población mundial con más de 60 años pasará de 900 millones a 2000 millones, lo que representa un aumento del $12 \%$ al $22 \%^{(1)}$. En Colombia, según el Departamento Administrativo Nacional de Estadística (DANE), el porcentaje de personas mayores de 60 años aumentó de 3,98\% en 1985 a $9,23 \%$ en $2018^{(2)}$. Además, se prevé que este aumento tendrá un profundo efecto en las sociedades tanto a nivel socioeconómico y cultural como en lo concerniente a las políticas de los sistemas de salud y protección social a nivel global.

Durante el envejecimiento se presentan cambios anatómicos, fisiológicos y funcionales que se reflejan tanto en la conducta como en el desempeño e independencia en la realización de tareas cotidianas, por ejemplo subir las escaleras, levantarse de una silla o caminar grandes distancias ${ }^{(3)}$. Una de las principales alteraciones que suceden durante este proceso es la sarcopenia, un fenómeno que involucra la pérdida progresiva del rendimiento físico y la reducción de la masa y la fuerza muscular, lo que se traduce en una mayor vulnerabilidad del paciente y representa mayores riesgos de complicaciones posoperatorias e internación prolongada en aquellos con otras enfermedades de base ${ }^{(4)}$. La prevalencia de la sarcopenia es de un $13 \%$ a $24 \%$ en personas entre 65 a 70 años, y se estima que puede llegar a un 50 $\%$ en personas mayores de 80 años ${ }^{(5)}$.

En la actualidad, cada vez son más los pacientes mayores de 60 años que por diversas razones deben someterse a cirugías de grande y mediana complejidad en los hospitales de todo el mundo; por lo tanto, se hacen necesarias medidas y/o protocolos de atención que disminuyan el riesgo de complicaciones posoperatorias y aceleren la recuperación del paciente, optimizando su salud ${ }^{(6)}$. El objetivo de este artículo de revisión es resaltar la importancia clínica de diagnosticar y tratar oportunamente las señales de sarcopenia en el paciente

\footnotetext{
Centro Universitário de Várzea Grande, Faculdade de Medicina. Várzea Grande, Mato Grosso, Brasil.

3 Faculdade de Medicina da Universidade Federal de Mato Grosso. Mato Grosso, Brasil.
}

quirúrgico al incentivar la implementación de programas multimodales que, al ser incorporados al plano de atención médica, permitan, a su vez, una recuperación más rápida y disminuyan el riesgo de complicaciones y mortalidad. Para la elaboración de este estudio se realizó una búsqueda no sistemática de artículos relevantes en español, inglés y portugués, utilizando las palabras clave "Sarcopenia", "Cirugía General", "Atención Perioperativa", "Fragilidad" y "Ejercicio físico". Las bases de datos consultadas fueron Medline, PubMed, Scielo y Google académico; se incluyeron ensayos clínicos, revisiones sistemáticas y metaanálisis publicados desde 1997 hasta 2020.

\section{DEFINICIÓN E HISTORIA DE LA SARCOPENIA}

La palabra sarcopenia proviene de la unión de dos palabras griegas: sarx, que significa "carne", y penia, que significa "pérdida". Este término fue utilizado inicialmente por Rosenberg para describir el proceso de pérdida de músculo que acontece con el envejecimiento ${ }^{(7)}$. La sarcopenia es una enfermedad muscular progresiva y generalizada que se caracteriza por una disminución de la fuerza muscular, la masa muscular y un deterioro en el desempeño físico, lo cual incrementa el riesgo de sufrir accidentes como fracturas y caídas y afecta la movilidad física, la realización de actividades diarias y la calidad de vida ${ }^{(8)}$. En 2016 la sarcopenia se incluyó como enfermedad en la Clasificación Internacional de Enfermedades y Problemas Relacionados con la Salud (CIE) de la OMS, con el código ICD-10-CM (M62.84), donde se reconoció como una condición geriátrica importante ${ }^{(9)}$.

Durante los últimos años este concepto ha venido tomando mayor fuerza entre profesionales de diferentes especialidades médicas como resultado de la necesidad de avanzar en la identificación y el cuidado de personas en riesgo y con sarcopenia. En el 2018 el European Working Group on Sarcopenia in Older People (EWGSOP), basado en investigaciones científicas, actualizó los principales criterios para la definición y el diagnóstico de la sarcopenia ${ }^{(4)}$. De este consenso se con- 
cluyeron 4 puntos importantes para ayudar en la prevención y el tratamiento de dicha enfermedad. El primero de ellos hace énfasis en que el desarrollo de la sarcopenia no solo se da en el envejecimiento, sino que también puede comenzar de forma más temprana en la vida de una persona, ya que las causas de dicho desorden muscular son múltiples y van más allá del envejecimiento. Factores como condiciones inflamatorias, hormonales, desórdenes neurológicos, enfermedades de base, falta de actividad física, sedentarismo, malnutrición o desórdenes alimenticios asociados con la anorexia o la obesidad pueden contribuir al deterioro muscular y posterior desarrollo de la sarcopenia. El segundo habla sobre el mayor enfoque brindado a la baja fuerza muscular como determinante principal en la identificación de la sarcopenia por encima de la baja masa muscular, lo cual se espera facilite la identificación en la práctica clínica. Este punto se relaciona con el tercero, ya que este afirma que la sarcopenia asociada con la baja cantidad y calidad muscular es técnicamente más difícil de medir y requiere de equipos especializados, por lo que resulta más dispendioso a la hora de evaluar y diagnosticar a los pacientes en la práctica clínica. Finalmente, en el último punto, el EWGSOP resalta la importancia de brindar una justificación más clara y práctica de las medidas y puntos de corte relevantes para el diagnóstico de la sarcopenia, ya que durante muchos años esta enfermedad fue tratada de forma inadecuada en la rutina hospitalaria debido a la complejidad de determinar cómo y qué variables medir, y qué puntos de corte considerar para el diagnóstico y tratamiento ${ }^{(4)}$.

\section{IMPORTANCIA DE LA EVALUACIÓN NUTRICIONAL Y FUNCIONAL EN PACIENTES QUIRÚRGICOS}

El proceso de envejecimiento compromete la estructura, funcionalidad, calidad y organización del músculo esquelético. A partir de la tercera década de vida se comienza a perder masa muscular a razón de $1 \%$ de músculo por año, lo cual puede incrementar hasta un $30 \%$-50\% alrededor de los 80 años ${ }^{(10,11)}$.

Gran parte de los pacientes mayores que van a pasar por algún procedimiento quirúrgico presentan no solo fragilidad y sarcopenia al ingreso médico, sino también otras comorbilidades existentes, como desnutrición, diabetes, hipertensión, depresión, enfermedades coronarias y/o pulmonares, cáncer y desórdenes neurológicos, que pueden comprometer aún más su estado nutricional y su recuperación posterior ${ }^{(12)}$. Los procedimientos quirúrgicos conllevan un trauma operatorio que implica una respuesta sistémica, la cual se asocia con alteraciones metabólicas y hormonales que, en pacientes con un estado nutricional comprometido y baja reserva muscular, pueden exacerbarse comprometiendo la evolución posoperatoria, la capacidad funcional y la calidad de vida a corto y largo plazo ${ }^{(13)}$.

$\mathrm{Al}$ verificar la relación entre las cirugías y la función muscular, Sierra y colaboradores ${ }^{(14)}$ encontraron que los procedimientos quirúrgicos disminuyen la función muscular posoperatoria de pacientes oncológicos sometidos a operaciones de gran complejidad, independientemente del riesgo nutricional. De hecho, otros estudios como el realizado por Bragagnolo y colaboradores $^{(15)}$ también han demostrado la importancia de la función muscular, al relacionar una baja fuerza muscular preoperatoria con una mayor mortalidad posoperatoria en pacientes sometidos a grandes cirugías. Así mismo, diferentes estudios han mostrado que pacientes con riesgo nutricional y déficit funcional al momento del ingreso médico tienen un mayor riesgo de presentar complicaciones infecciosas en el posoperatorio, mayor tiempo de internación, retraso en la cicatrización de heridas, mayor incidencia de úlceras por presión, readmisiones más frecuentes y mayor tiempo de recuperación general, lo cual, a su vez, también representa un incremento en los costos de salud hospitalaria ${ }^{(16-20)}$.

De acuerdo con lo anterior, es fundamental realizar una evaluación preoperatoria que involucre aspectos clínicos, nutricionales y funcionales del paciente. Esto es importante no solo para realizar un diagnóstico integral e interdisciplinario, sino también para desarrollar intervenciones y tratamientos que ayuden a disminuir el riesgo de complicaciones perioperatorias y permitan, al mismo tiempo, una recuperación satisfactoria y rápida en los pacientes ${ }^{(21)}$.

La identificación de pacientes con posible sarcopenia en la práctica clínica se realiza a través de diferentes pruebas y métodos, cuya elección y aplicación dependen de la condición médica y funcional de cada paciente. Una de las herramientas recomendada y validada por el EWGSOP es el cuestionario Strength, Assistance with walking, Rise from a chair, Climb stairs and Falls (SARC-F), que incluye preguntas sobre 5 componentes claves en la identificación de signos y características de riesgo de sarcopenia: fuerza, asistencia para caminar, levantarse de una silla, subir escaleras e historial de caídas (Tabla 1$)^{(22)}$. 
Tabla 1. Cuestionario SARC-F para evaluar el riesgo de sarcopenia ${ }^{(22)}$

\begin{tabular}{|l|l|l|}
\hline \multicolumn{1}{|c|}{ Componente } & \multicolumn{1}{|c|}{ Pregunta } & \multicolumn{1}{c|}{ Puntuación } \\
\hline Fuerza & ¿Cuánta dificultad tiene para levantar y cargar 4,5 kg? & $\begin{array}{l}\text { 0: Ninguna } \\
\text { 1: Alguna } \\
\text { 2: Mucha/ incapaz }\end{array}$ \\
\hline Asistencia para caminar & ¿Cuánta dificultad tiene para cruzar una habitación? & $\begin{array}{l}\text { 0: Ninguna } \\
\text { 1: Alguna } \\
\text { 2: Mucha/ requiere ayuda/ incapaz }\end{array}$ \\
\hline Levantarse de una silla & $\begin{array}{l}\text { ¿Cuánta dificultad tiene para trasladarse desde una silla/ } \\
\text { cama? }\end{array}$ & $\begin{array}{l}\text { 0: Ninguna } \\
\text { 1: Alguna } \\
\text { 2: Mucha/ es incapaz sin ayuda }\end{array}$ \\
\hline Subir escaleras & $\begin{array}{l}\text { ¿Cuánta dificultad tiene para subir un tramo de diez } \\
\text { escalas? }\end{array}$ & $\begin{array}{l}\text { 0: Ninguna } \\
\text { 1: Alguna } \\
\text { 2: Mucha/ incapaz }\end{array}$ \\
\hline Caídas & ¿Cuántas veces se ha caído en el último año? & $\begin{array}{l}\text { 0: Ninguna } \\
\text { 1: 1-3 veces } \\
\text { 2: } \text { o más veces }\end{array}$ \\
\hline
\end{tabular}

Traducido y adaptado de ${ }^{(22)}$.

Para cada componente, los pacientes son evaluados con 0,1 o 2 puntos (0 representa ninguna dificultad, 1 supone alguna dificultad y 2 mucha dificultad o incapacidad). Ahora bien, si la sumatoria final del paciente registra entre 0-3 puntos significa que no tiene riesgo de sarcopenia; pero si por el contrario la puntuación final está entre 4-10 puntos, el paciente tiene riesgo de sarcopenia y se debe continuar con la evaluación empleando otras herramientas como la dinamometría o el "Chair stand test", para confirmar el diagnóstico y la severidad ${ }^{(4)}$.

El estudio realizado por Malmstrom y colaboradores $^{(23)}$,en el cual se investigó la utilidad del SARC-F en 3 grandes estudios (the African American Health [AAH] study, Baltimore Longitudinal Study of Aging [BLSA], y National Health and Nutrition Examination Survey [NHANES]), demostró que dicho cuestionario fue válido y consistente para detectar a personas en riesgo de obtener resultados adversos por causa de la sarcopenia. Con base en estos hallazgos, puede sugerirse que el SARC-F sirve como herramienta clínica rápida y de bajo costo para aplicarse durante la atención primaria en la identificación de personas con debilidad muscular y con riesgo de sarcopenia. En este sentido, un metaanálisis reciente concluyó que sujetos sarcopénicos tienen un mayor riesgo de mortalidad y que este efecto es aún más probable en personas mayores de 79 años, en la medida en que este se relaciona con un declive funcio- nal, una mayor tasa de caídas y una mayor incidencia de hospitalizaciones $^{(24)}$.

Según el EWGSOP, la medición de la fuerza de agarre a través de la dinamometría es otro de los parámetros útiles para diagnosticar sarcopenia; su fácil aplicación en la práctica clínica la convierte en una medida confiable y segura para predecir resultados negativos en los pacientes y ser utilizada en entornos clínicos especializados $^{(4)}$. Esta medida, además de brindar información sobre la fuerza muscular, permite conocer indirectamente información sobre el estado nutricional, la función física, la masa muscular y el estado de salud general del paciente ${ }^{(23)}$. Diversos estudios han relacionado la baja fuerza de agarre con un mayor tiempo de internación hospitalaria ${ }^{(25,26)}$, mayor riesgo de mortalidad ${ }^{(27)}$, desarrollo de enfermedades cardiovasculares ${ }^{(28)}$, limitaciones funcionales y peor calidad de vida en general ${ }^{(29)}$.

\section{PROGRAMAS MULTIMODALES EN EL PACIENTE QUIRÚRGICO CON SARCOPENIA}

Actualmente, el manejo terapéutico de la sarcopenia se enfoca en intervenciones nutricionales y hormonales (con testosterona, estrógenos, hormona de crecimiento humano y otros anabolizantes), modulación de la función inmunitaria e intervenciones como el ejercicio físico de resistencia ${ }^{(30)}$. Sin embargo, la aplicación de 


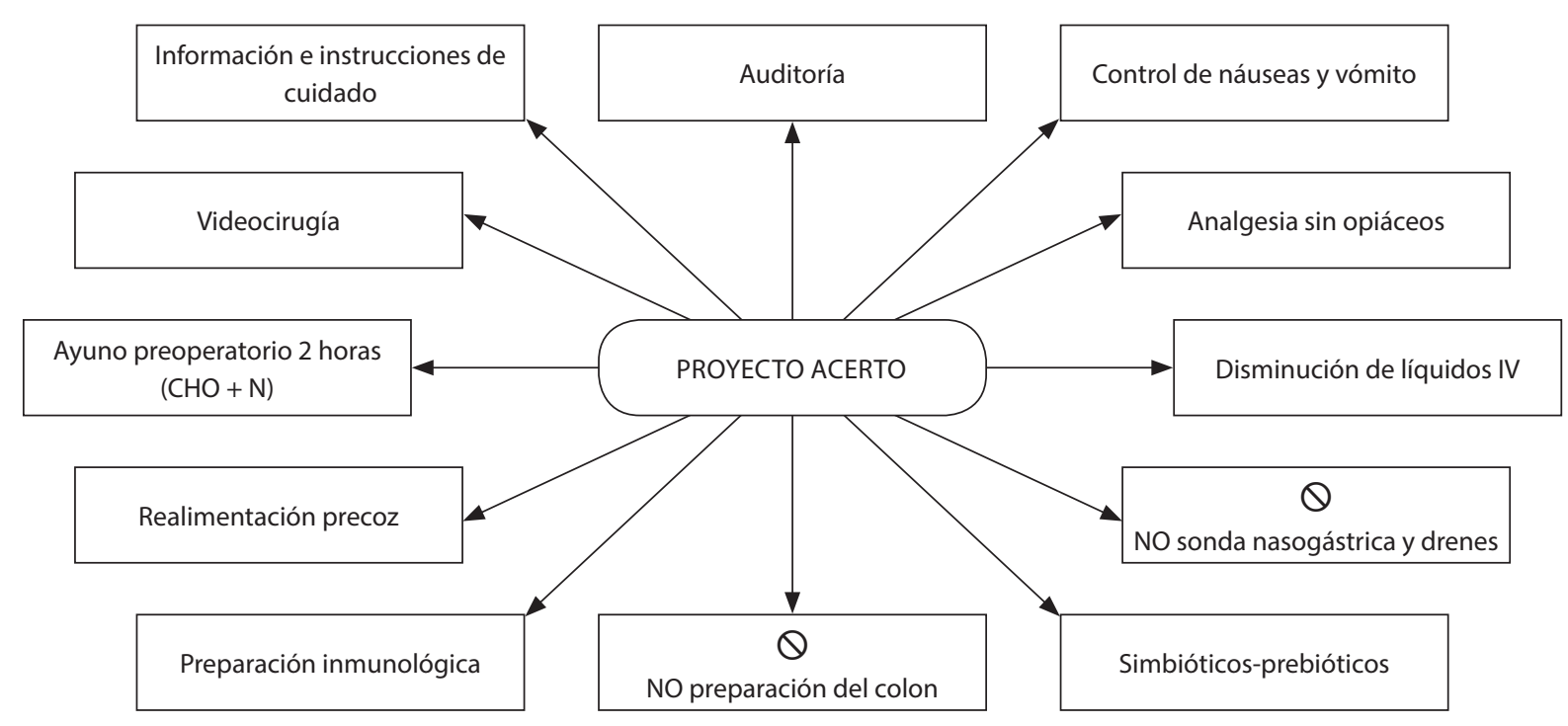

Figura 1. Principales conductas del proyecto ACERTO. Tomado de ${ }^{(33)}$

una u otra terapia está sujeta a la evaluación de las condiciones particulares de cada paciente, tales como su estado de salud, funcionalidad y enfermedades de base.

Los adultos mayores candidatos a cirugías de grande y mediana complejidad generalmente presentan diferentes enfermedades y condiciones de salud que coexisten; es decir, un mismo paciente puede padecer cáncer, pero a su vez presentar hipertensión y estar sarcopénico, por lo cual las intervenciones tanto médicas como nutricionales deben estar diseñadas para apuntar a múltiples condiciones ${ }^{(31)}$.

En este sentido, desde hace varios años se vienen construyendo y consolidando a nivel mundial los programas "fast-track" o Enhanced Recovery After Surgery (ERAS), los cuales hacen referencia a un conjunto de medidas y estrategias perioperatorias basadas en evidencia científica, cuyo objetivo es disminuir el estrés metabólico originado por la intervención quirúrgica y mejorar significativamente la recuperación y la funcionalidad del paciente; esto, a su vez, también reduce costos hospitalarios en el sistema de salud pública ${ }^{(32)}$. Un ejemplo de estos programas multimodales es el protocolo de Aceleração da Recuperação Total pós-operatória (ACERTO) en América Latina, el cual, a través de la implementación de una serie de conductas y prácticas perioperatorias, ha mostrado resultados satisfactorios en la disminución del tiempo de internación, número de complicaciones posoperatorias y mortalidad (Figura 1) $)^{(33)}$.
Los procedimientos quirúrgicos se comparan con una maratón, en la cual la persona que va a participar necesita una preparación previa para así tener un buen desempeño durante la carrera y una buena recuperación posterior. De igual forma sucede con las operaciones de grande y mediana complejidad; los pacientes necesitan preparase a través de medidas aplicadas en el preoperatorio para mejorar el desempeño funcional, reducir el riesgo de complicaciones, el riesgo de mortalidad y acelerar la recuperación posoperatoria. En este contexto surge la prehabilitación, una estrategia multimodal que busca optimizar la salud del paciente gracias a la implementación de medidas que envuelven ejercicio físico, aspectos nutricionales, soporte psicológico e interrupción de comportamientos negativos para la salud ${ }^{(34)}$.

El estudio realizado por Gillis y colaboradores ${ }^{(35)}$ con pacientes entre los 66-68 años demostró que la implementación de un programa de prehabilitación en el período preoperatorio logró disminuir la pérdida de masa magra posoperatoria y consiguió modular la respuesta al trauma operatorio en los pacientes con cáncer colorrectal. De acuerdo con Barberan-García y colaboradores $^{(36)}$, la prehabilitación debe ser una intervención central dentro del curso preoperatorio de pacientes con alto riesgo sometidos a cirugías electivas de gran complejidad. Los resultados de este estudio mostraron cómo a través de la implementación de un programa de prehabilitación personalizado durante 6 semanas 
es posible reducir el número de pacientes con complicaciones posoperatorias hasta en un $51 \%$, y mejorar además su capacidad aeróbica. Sucesivos estudios han demostrado también estos resultados ${ }^{(37,38)}$.

A corto plazo, la aplicación de los diversos programas de prehabilitación permite mejorar la capacidad física y funcional del paciente, y acelerar su recuperación y proceso de mejoría. De igual forma, en aquellos pacientes con enfermedades cardiovasculares permite tener una mejora en la función cardíaca y en la preparación cardiorrespiratoria, lo que permite al paciente retornar más rápidamente a su estado óptimo de salud física y psicológica ${ }^{(34)}$. A largo plazo, la prehabilitación también ayuda a disminuir la ansiedad, la depresión posoperatoria y la baja autoestima.

Los programas de prehabilitación que existen actualmente varían de acuerdo con las diferentes organizaciones, por lo cual aún no existe un consenso que determine el ejercicio y las intervenciones en nutrición a ser prescritos. Dichos programas necesitan ser personalizados y ofrecidos por un equipo interdisciplinario, además de tener en cuenta el tipo de cirugía y las condiciones de salud particulares de cada persona. Si bien, la prehabilitación disminuye el tiempo de internación, el riesgo de complicaciones posoperatorias, el número de reinternaciones y mejora la capacidad funcional, son necesarios más estudios que analicen qué tipo de pacientes se benefician más de estos programas y si su adopción a gran escala es rentable y sostenible a largo plazo dentro de la incorporación en la rutina hospitalaria ${ }^{(36)}$.

\section{CONCLUSIÓN}

La sarcopenia es un síndrome cuya prevalencia va en aumento; es de vital importancia realizar un diagnóstico clínico y un tratamiento oportuno con el fin de prevenir complicaciones y un mayor deterioro de la capacidad funcional del paciente. El período preoperatorio es clave para optimizar la salud física e implementar medidas que promuevan la recuperación del paciente, mostrando mejores resultados y la disminución de morbimortalidad postoperatoria. Creemos que medidas como la prehabilitación pueden beneficiar tanto a pacientes sarcopénicos candidatos a cirugías como a aquellos ya internados, al mejorar los resultados posoperatorios, la capacidad funcional y la calidad de vida en general.

\section{Financiamiento}

El presente estudio no tuvo financiación.

\section{Conflicto de intereses}

No declaran.

\section{Declaración de autoría}

Todos los autores revisaron el artículo y validaron su versión final.

\section{Referencias bibliográficas}

1. Organización Mundial de la Salud. Informe Mundial sobre el envejecimiento y la salud. [Internet] [Fecha de consulta: 1 de julio de 2020]. Organización Mundial de la Salud; 2015. Disponible en: https://apps.who.int/iris/bitstream/handle/10665/186466/9789240694873_spa.pdf;jsessionid=9B F254CC83A7D3C55E3FC7C5B4B370BC? sequence $=1$.

2. Ministerio de Salud y Protección Social. Envejecimiento demográfico. Colombia 1951-2020 dinámica demográfica y estructuras poblacionales. [Internet]. Ministerio de Salud y Protección Social; 2013. Disponible en: https://www.minsalud.gov. $\mathrm{co} /$ sites/rid/Lists/BibliotecaDigital/RIDE/DE/PS/ Envejecimiento-demografico-Colombia-1951-2020.pdf.

3. Garcia PA, Dias JMD, Rocha ASS, et al. Relação da capacidade funcional, força e massa muscular de idosos com osteopenia e osteoporose. Fisioter Pesq. 2015;22(2):126-32. doi: $10.590 / 1809-2950 / 13154522022015$.

4. Cruz-Jentoft AJ, Bahat G, Bauer J, et al. Sarcopenia: revised European consensus on definition and diagnosis. Age and ageing. 2018;48(1):16-31. doi: 10.1093/ageing/afy169.

5. Parra B, Matos LN, Ferrer R, et al. SARCPRO: Proposta de protocolo para sarcopenia em pacientes internados. Braspen J. 2019;34(1):58-63.

6. Chapela S, Martinuzzi A. Pérdida de masa muscular en el paciente críticamente enfermo: ¿Caquexia, sarcopenia y/o atrofia? Impacto en la respuesta terapéutica y la supervivencia. Rev Cuba aliment nutr. 2018;28(2):393-416.

7. Rosenberg IH. Sarcopenia: origins and clinical relevance. J Nutr.1997;127(5 Suppl):990S-991S. doi: 10.1093/ jn/127.5.990S.

8. Santilli V, Bernetti A, Mangone M, et al. Clinical definition of sarcopenia. Clin Cases Miner Bone Metab. 2014;11(3):177-80.

9. Cao Li, Morley JE. Sarcopenia is recognized as an independent condition by an international classification of disease, tenth revision, clinical modification (ICD-10-CM) code. J Am Med Dir Assoc. 2016;17(8):675-77. doi: 10.1016/j. jamda.2016.06.001.

10. Ali S, Garcia JM. Sarcopenia, cachexia and agind: diagnosis, mechanisms and therapeutic options. Gerontology. 2014;60(4):294-304. doi: 10.1159/000356760.

11. Pinzón-Ríos ID. Pérdida de masa muscular inducida por el envejecimiento. Rev Cienc Salud Bogotá. 2019;17(2):223-44. doi: 10.12804/revistas.urosario.edu.co/revsalud/a.7925. 
12. Sánchez-Castellano C, Martín-Aragón S, Vaquero-Pinto N. Prevalencia de sarcopenia y características de los sarcopénicos en pacientes mayores de 80 años ingresados por fractura de cadera. Nutr Hosp. 2019;36(4):813-18. doi: 10.20960/ nh.02607.

13. Desborough J. The stress response to trauma and surgery. $\mathrm{Br} \mathrm{J}$ Anaesth. 2000;85(1):109-17. doi: 10.1093/bja/85.1.109.

14. Sierra JC, Dock Nascimento DB, Behne TEG, et al. Cirurgia oncológica de grande porte reduz a função muscular de pacientes com e sem risco nutricional. Rev Col Bras Cir. 2020;47:e20202470. doi: 10.1590/0100-6991e-20202470.

15. Bragagnolo R, Caporossi FS, Dock-Nascimento DB, et al. Handgrip strength and adductor pollicis muscle thickness as predictors of postoperative complications after major operations of the gastrointestinal tract. e-SPEN, Eur e-J Clin Nutr Metabol.2011;6(1):e21-e26. doi: https://doi.org/10.1016/j. eclnm.2010.11.001.

16. Paredes-Terrones X, Pérez-Agüero C, Runzer-Colmenares FM, et al. Factores asociados a complicaciones quirúrgicas en pacientes adultos mayores con neoplasias gastrointestinales del Centro Médico Naval. Horizonte Médico (Lima). 2020;20(1):45-53. doi: 10.24265/horizmed.2020.v20n1.07.

17. Gariballa S, Alessa A. Sarcopenia: prevalence and prognostic significance in hospitalized patients. Clin Nutr. 2013;32(5):772-76. doi: 10.1016/j.clnu.2013.01.010.

18. Lieffers JR, Bathe OF, Fassbender K, et al. Sarcopenia is associated with postoperative infection and delayed recovery from colorectal cancer resection surgery. $\mathrm{Br} \mathrm{J}$ Cancer. 2012;107(6):931-36. doi: 10.1038/bjc.2012.350.

19. Menezes CS, Fortes RC. Estado nutricional y evolución clínica de ancianos en terapia nutricional enteral domiciliaria: cohorte retrospectiva. Rev Latino-Am Enfermagem. 2019;27:e3198. doi: 10.1590/1518-8345.2837.3198.

20. Somoza EMZ, Álvarez VF, Porbén SS. Sobre las interrelaciones entre la sarcopenia, envejecimiento y nutrición. Rev Cuba aliment nutr. 2018;28(1):152-76.

21. Kunze S. Evaluación preoperatoria en el siglo XXI. Rev méd Clín Las Condes. 2017;28(5):661-70.

22. Morley JE, Little MO, Berg-Weger M. Rapid geriatric assessment: a tool for primary care physicians. J Am Med Dir Assoc. 2017;18(3):195-99. doi: 10.1016/j. jamda.2016.11.017.

23. Malmstrom TK, Miller DK, Simonsick EM, et al. SARC-F: a symptom score to predict persons with sarcopenia at risk for poor functional outcomes. J Cachexia Sarcopenia Muscle. 2016;7(1):28-36. doi: 10.1002/jcsm.12048.

24. Beaudart C, Zaaria M, Pasleau F, et al. Health Outcomes of Sarcopenia: A Systematic Review and Meta-Analysis. PloS one. 2017;12(1):e0169548. doi: 10.1371/journal. pone.0169548.

25. Mendes J, Alves P, Amaral TF. Comparison of nutritional status assessment parameters in predicting length of hospital stay in cancer patients. Clin Nutr. 2014;33(3):466-70. doi: 10.1016/j.clnu.2013.06.016.
26. Shah M, Shah S, Shah D. Hand-Grip Strength as an Effective Predictor of Surgical Outcomes in Malignancy. Indian J Surg. 2019;81(1):7-10. doi: 10.1186/1749-8090-6-98.

27. Yates T, Zaccardi F, Dhalwani NN, et al. Association of walking pace and handgrip strength with all-cause, cardiovascular, and cancer mortality: a UK Biobank observational study. Eur Heart J. 2017;38(43):3232-40. doi: 10.1093/eurheartj/ehx449.

28. Leong DP, Teo KK, Rangarajan S, et al. Prognostic value of grip strength: findings from the Prospective Urban Rural Epidemiology (PURE) study. The Lancet. 2015;386(9990):266-73. doi: 10.1016/S0140-6736(14) 62000-6.

29. McNicholl T, Curtis L, Dubin JA, et al. Handgrip strength predicts length of stay and quality of life in and out of hospital. Clin Nutr. 2020;39(8):2501-09. doi: 10.1016/j. clnu.2019.11.006.

30. Cortés WAG, Fernández FEM, Sanmiguel LCO. Sarcopenia, una patología nueva que impacta a la vejez. Revista Colombiana de Endocrinología, Diabetes \& Metabolismo. 2018;5(1):28-36.

31. Samper-Ternent R, Reyes-Ortiz C, Ottenbacher KJ, et al. Frailty and sarcopenia in Bogotá: results from the SABE Bogotá Study. Aging Clin Exp Res. 2017;29(2): 265-72. doi: 10.1007/s40520-016-0561-2.

32. Sánchez P, Vargas E. Superación de barreras en la implementación de los protocolos ERAS: aspectos nutricionales. Rev Nutr Clin Metab. 2020;3(1):100-107. doi: 10.35454/rncm. v3n1.022.

33. de Aguilar-Nascimento JE, Sierra JC, Dock Nascimento DB. El Proyecto ACERTO: un protocolo multimodal barato y eficaz para América Latina. Rev Nutr Clin Metab. 2020;3(1):9199. doi: 10.35454/rncm.v3n1.018.

34. Gonçalves CG, Groth AK. Pré-habilitação: como preparar nossos pacientes para cirurgias abdominais eletivas de maior porte? Rev Col Bras Cir. 2019;46(5):e20192267. doi: 10.1590/0100-6991e-20192267.

35. Gillis C, Fenton TR, Sajobi TT, et al. Trimodal prehabilitation for colorectal surgery attenuates post-surgical losses in lean body mass: a pooled analysis of randomized controlled trials. Clin Nutr. 2019;38(3):1053-60. doi: 10.1016/j. clnu.2018.06.982.

36. Barberan-García A, Ubre $\mathrm{M}$, Roca J, et al. Personalised prehabilitation in high-risk patients undergoing elective major abdominal surgery: a randomized blinded controlled trial. Ann Surg. 2018;267(1):50-6. doi: 10.1097/ SLA.0000000000002293.

37. López LM, Llovera AP, Serra-Aracil X, et al. Estudio observacional prospectivo unicéntrico sobre el efecto de la prehabilitación trimodal en cirugía colorrectal. Cirugía Española. 2020;98(10):605-11 doi: 10.1016/j.ciresp.2020.03.023.

38. Minnella EM, Awasthi R, Gillis C, et al. Patients with poor baseline walking capacity are most likely to improve their functional status with multimodal prehabilitation. Surgery. 2016;160(4):1070-9. doi: 10.1016/j.surg.2016.05.036. 